\title{
Anabases
}

ANABASES Traditions et réceptions de l'Antiquité

11 | 2010

Varia

\section{Erreurs de traduction volontaires et paresse du contresens}

Pierre-Emmanuel Dauzat

\section{(2) OpenEdition}

1 Journals

Édition électronique

URL : http://journals.openedition.org/anabases/826

DOI : 10.4000/anabases.826

ISSN : 2256-9421

Éditeur

E.R.A.S.M.E.

Édition imprimée

Date de publication : 1 mars 2010

ISSN : 1774-4296

\section{Référence électronique}

Pierre-Emmanuel Dauzat, "Erreurs de traduction volontaires et paresse du contresens », Anabases [En ligne], 11 | 2010, mis en ligne le 01 mars 2013, consulté le 14 novembre 2019. URL : http://

journals.openedition.org/anabases/826 ; DOI : 10.4000/anabases.826

(c) Anabases 


\section{Erreurs de traduction volontaires et paresse du contresens}

Pierre-Emmanuel Dauzat

Les eRREURS DE TRADUCtion ONT MAUVAISE RÉPUTATION. On les dénonce et on les pourchasse au point de vouloir, à l'exemple du philosophe Jacques d'Hondt, les « qualifier juridiquement ». "J'enverrais tous ces Messieurs les traducteurs en Sibérie pour manque de patriotisme et légèreté », rageait déjà le doux Tchékhov. Ce faisant, on oublie trop souvent que la traduction n'est pas une discipline autonome, qu'elle est souvent en position ancillaire, et que les erreurs commises, notamment dans le domaine de la théologie, mais aussi en philosophie ou en histoire, font jurisprudence et créent des précédents ${ }^{1}$. L'erreur involontaire devient alors volontaire, et la correction de l'erreur de traduction ne change rien à ses conséquences. Ces quelques observations de traducteur praticien sur l'histoire de la traduction n'ont d'autre but que de donner un aperçu de la place que devrait occuper la réflexion sur l'erreur dans l'histoire et la théorie de la traduction pour remettre en cause l'idée reçue que l'erreur de traduction est, "pis qu'un crime, une faute ".

Tous les traducteurs ou auteurs de contresens n'ont pas les obligeances ni les élégances de Ronsard, dans l' Hymne à la mort:

Adonc la Mort se sied dessus leur blanche teste

Qui demande sa debte, et la veut avoir preste;

Ou bien si quelques jours, pour leur faire plaisir,

Les souffre dans le lict languir, tout à loisir,

Si est-ce que soudain, après l'usure grande

D'yeux, de braz, ou de piedz, sa debte redemande,

Et veut avec l'usure avoir le principal.

1 Sur cette notion, voir P.-E. DAUZAT, "Jurisprudence(s) de la traduction ", Colloque de Toulon, 24-25 novembre 2005, in J.-J. SuEur, éd., Interpréter et traduire, Bruxelles, Bruylant, 2007, p. 217-231; et "L'expédient en traduction: du parler-en-langues au contresens volontaire", in P. Loraux et C. Énaudeau, La Méthode de l'expédient, Paris, Kimé, 2006, p. 165-180. 
S'inspirant de la traduction par Marsile Ficin de l'Axiochos du Pseudo-Platon, avec son suggestif astans super caput natura, il commence en effet par en donner une traduction littérale à contresens, avec l'image de " la mort assise sur la tête de ses débiteurs ", avant d'effacer ce contresens volontaire et de se faire traducteur plus orthodoxe en retraduisant, à quelques vers plus loin, la même formule, super caput, par "veut avec l'usure avoir le principal ". La reprise de la même formule traduite l'une à contresens, l'autre avec exactitude signe en effet le contresens volontaire. Qui lit le dernier vers cité ne saurait douter que Ronsard savait son latin et l'a bien traduit ${ }^{2}$. Ce faisant, Ronsard s'inscrit dans la droite ligne de son confrère de la Pléiade, à savoir Du Bellay et sa Deffence et illustration de la langue françoyse de 1549, qui tout en forgeant par paronomase le faux couple italien traduttore-traditore, revendique le " droit à l'erreur " pour éviter le psittacisme: bref, il faut " oser faire des traductions erronées ». François Rigolot observe: "En termes derridiens on pourrait parler d'une théorie de l'erratum puisque l'incorrection y est la condition sine qua non de la production de sens. En termes judéochrétiens, la transgression se trouve rachetée par une économie sotériologique: felix $c u l p a^{3}$. " Autrement dit, Ronsard sait traduire, mais il revendique le droit à l'erreur pour faire naître un autre Cupidon de ses noces avec la philologie.

Mais la double traduction à la Ronsard, avec son contresens volontaire, n'est pas sans surprises et risque de déboucher sur des " faux contresens " et non plus un " contresens volontaire ". Michel Zink l'a bien vu à propos du "faux Haimon " et de sa référence à Matthieu 21,1-9 sur l'arrivée à Bephtague, qualifié de "rue " (vinculus), parce que sans murs, puis l'entrée à Jérusalem ${ }^{4}$ : In illo tempore, cum appropinquassent Hierosolymis et venissent Bephtague. L'auteur traduit par " rue » tout en se justifiant parce que, dit-il, certes tous les villages en Terre sainte sont fortifiés, ainsi que le précise Mat 21,2. Or, le pseudo-Haimon commet ensuite un contresens sur Mat 21,2, où Jésus dit à ses disciples: Ite est castellum, quod contra vos est. Le traducteur comprend que castellum désigne Jérusalem; peut-être s'autorise-t-il d'un commentaire de saint Jérôme: "... Misit duos discipulos, theôrêtikon kai ergastikon, id est, scientiam et operam, ut ingrederentur castellum. Dixitque eis: Ite in castellum quod contra vos est. Contra apostolos enim erat, nec jugum doctrinarum volebat accipere " (Comm. Ev. Mat., PM 26, 146-147). Le sermon est le seul à mentionner l'opposition des Juifs, laquelle ne peut être figurée que par Jérusalem. L’auteur, croyant préciser la pensée de saint Jérôme

2 J. CÉARD, "Ronsard, lecteur de l'Axiochos: une source inconnue de l'Hymne à la mort", in L. Zilli, éd., Parcours et rencontres. Mélanges de langue, d'histoire et de littérature françaises offerts à Enea Balmas, Paris, Klincksieck, 1993, tome 1, p. 105-115, ici p. 107.

3 F. Rigolot, L'Erreur de la Renaissance. Perspectives littéraires, Paris, Champion, 2002, p. 309-311. Cf. O.P. Norton, The Ideology and Langage of Translation in Renaissance France and their Humanist Antecedents, Genève, Droz, 1984, p. 292 sq.

4 M. ZinK, "Les 'rues' de la Terre promise (vicus: rue). Une bonne traduction du faux Haimon et un faux contresens sur saint Matthieu", Romania, 378-379, 1974, $\mathrm{n}^{\circ} 2-3$, p. 351-358. 
demeuré ambigu, commet un contresens, mais un contresens qui ne porte que sur l'interprétation allégorique du passage: «Le commentaire sur vinculus n'empêche pas l'auteur de comprendre la lettre de l'Évangile. [...] Sans doute aurait-il hésité à exposer concurremment deux sens contradictoires se rapportant l'un et l'autre à la lettre du texte sacré, ou deux interprétations allégoriques incompatibles. Mais se sentait-il contraint de rendre cohérents et compatibles deux niveaux de signification différents? Il semble bien que non. » Pour nous résumer, le pseudo-Haimon traduit deux fois castellum: la première fois, pour en déduire que Bephtague (litt. " maison de bouche ", désignant selon certains commentateurs les chiens de garde du seigneur qui rappellent les fidèles dans le droit chemin) est une place fortifiée; la seconde, l'appliquant à Jérusalem, pour dire que la ville sainte est murée contre le Christ.

Ces paramètres du contresens et de son indétermination relative étant définis, venons-en à l'étude concrète et à la possibilité d'appliquer ces notions à la critique des traductions. Dans son essai De la traduction (1635), Claude Gaspar Bachet de Meziriac, que Descartes admirait fort, s'en prend aux « Belles infidèles » et brave l'adoubement donné par Montaigne à la traduction de Plutarque par Amyot pour dresser une typologie des erreurs de sens en des termes qui rappellent La Manière de bien traduire d'Étienne Dolet (1540). «[...] Si quelqu'un aspire à la louange que mérite une fidèle traduction, il faut qu'il observe exactement ces trois points; qu'il n'ajoute rien à ce que dit son Auteur, qu'il n'en retranche rien, et qu'il n'y apporte aucun changement qui puisse en altérer le sens ${ }^{5}$." Pour mesurer la pertinence de ce modèle, apparemment indépassé $e^{6}$, arrêtons-nous sur la traduction en tous points excellente d'un maître en la matière, celle des Analectes de Confucius par Pierre Ryckmans, alias Simon Leys ${ }^{7}$ :

Ji Zicheng dit: "On est honnête homme par nature; à quoi bon la culture?" Zigong dit: "Monsieur, voilà un mot regrettable; ce qui est dit est dit: un attelage de quatre chevaux ne saurait le rattraper. La culture tient à la nature, la nature tient à la culture, comme sa bigarrure au tigre. [...]" (XII, 8).

Dans le corps du texte, rien ne signale l'omission, mais la note précise: "Voilà un mot regrettable: entre cette phrase et la suivante sont insérés les trois mots, jun zi ye, que j'ai omis dans ma traduction, ne pouvant trancher entre trois sens possibles: 1) Vous parlez en honnête homme, mais ce que vous avez dit est regrettable; 2) Ce que vous avez dit au sujet de l'honnête homme est regrettable; 3) Il est regrettable que vous ayez dit

5 Claude Gaspar BACHET De MeZiriac, De la traduction (1635), réédité avec une introduction de M. Ballard in Artois Presses Université/Presses de l'Université d'Ottawa, 1998, p. XXXIV sq. et 8.

6 Cf. L'Erreur en traduction, sous la direction de R. LAROSE, TTR. Traduction, terminologie, traduction. Études sur le texte et ses transformations, 2/2, 1989, qui montre que la problématique de l'erreur reste largement descriptive et peine à définir une théorie générale en raison du caractère téléologique de l'activité de traduction.

7 Confucius, Les Entretiens de Confucius, trad. et annoté par P. Ryckmans, préface d'Étiemble, Paris, Gallimard, 1987, p. 66-67 et note p. 149. 
cela; car quand un honnête homme a parlé, un attelage de quatre chevaux ne pourrait rattraper ses paroles. (Aujourd'hui les grammairiens considèrent que seule la lecture $\mathrm{n}^{\circ} 2$ est correcte, quoique la lecture $\mathrm{n}^{\circ} 3$ ne soit pas impossible. Selon nous la lecture $\mathrm{n}^{\circ} 1$ est à exclure; mais il faut pourtant remarquer que c'était celle de Zhu Xi. Évidemment, Zhu Xi n'était pas grammairien...). » Pour se refuser à traduire, le traducteur évoque l'ambiguïté, sans chercher à la rendre par une ambiguïté ni prendre le risque du contresens dans sa propre langue qu'il impute à Zhu Xi, lettré de la dynastie Song, lequel joua pourtant un rôle essentiel dans la constitution du canon des classiques chinois.

Après le non possumus du traducteur qui se refuse à trancher et émende, vient l'ajout qui explicite - comme sa bigarrure tient au tigre: "Ces mots ne sont pas dans le texte, mais ils explicitent le double sens de wen: "culture" au sens dérivé, mais "bigarrure [du pelage d'un animal]" au sens premier. " On retrouve donc ici, pleinement assumés, les trois péchés dénoncés par Bachet de Meziriac en 1635, sous la plume d'un traducteur émérite, il est vrai tenté par l'éloge du contresens ${ }^{8}$. Ici, le traducteur va à contresens en refusant de traduire puis en ajoutant après avoir omis ${ }^{9}$. L'examen d'un autre texte, le Testimonium de Flavius Josèphe, montrerait à l'inverse comment à trop vouloir traduire un texte apparemment simple, on peut s'exposer au contresens inavoué en faisant passer une idéologie sous couvert de traduction ${ }^{10}$. L'une des conclusions les plus claires est que, quand on traduit, on fait toujours autre chose que traduire, et les décisions de sens opérées ne relèvent pas toutes de la linguistique et de la traductologie - l'exégèse, voire l'acte de foi, suppléant aux lacunes du texte. Serge Bardet conclut: "Bref, un traducteur ne peut pas feindre de croire que son lecteur et lui vont conjointement s'abstraire du passé de la question. " Autrement dit, il n'y a pas une première étape qui serait l'établissement d'un texte et sa traduction, puis une seconde qui serait celle de son interprétation: celle-ci fait partie intégrante de celle-là. Même si certaines décisions jurisprudentielles s'imposent pour éviter de tirer des conclusions avant même que la recherche historique n'ait été accomplie. Il faut donc compter avec la paresse du contresens, qui n'est autre que le fait accompli. L'apparente simplicité du texte de Flavius Josèphe met en évidence un problème majeur théorisé par le philosophe W. Quine, celui de "l'indétermination " de la traduction et de l'indécidabilité du sens,

8 S. LeYs, "Traduction (théorie et pratique)", in LeYs, L'Ange et le cachalot, Paris, Seuil, 1998, p. 135-204.

9 "Omettre ou ajouter, abréger ou paraphraser, ce n'est pas traduire ", dira M. Desaintange dans la préface à sa traduction des Métamorphoses d'Ovide, Paris, 1808, p. XLIX, qui admettait pourtant qu'on puisse " développer par des expressions interprétatives » sans manquer au devoir du traducteur.

10 Cf. S. BARDET, Le Testimonium flavianum. Examen historique. Considérations historiographiques, préface de P. Geoltrain, Paris, Cerf, 2002 et "Le Testimonium Flavianum, intraduisible et illimité? (Flavius Josèphe, Antiquités judaïques XVIII, \$ 63-64)", in C. Mimouni et I. Uttern-Weité, éd., Pierre Geoltrain ou comment " faire l'histoire des religions"? Le chantier des "origines", les méthodes du doute et la conversation contemporaine entre les disciplines, Turnhout, Brepols, 2006. 
même dans des cas apparemment limpides. Les inflexions virtuelles sont innombrables, suivant les hypothèses de départ, et dès lors que l'exégèse ou la foi vole au secours de la philologie, le "forcènement du sens ", pour parler comme Derrida à propos d'Artaud, peut cesser d'être involontaire pour être volontaire.

Il n'est pas innocent que la traduction du nom de Judas " l'Iscariote ", dans le Nouveau Testament, se heurte aux mêmes apories, dirimantes sauf pour des traducteurs trop pressés... ou qui acceptent le secours de l'exégèse ou de l'Esprit-saint, comme le suggère Marguerite Harl dans La Revanche d'Érasme ${ }^{11}$. Le nom de l'Iscariote, en effet, est de ces hapax dont la traduction se refuse, ou devrait se refuser, à toute tentative d'annexion, pour la simple raison qu'on ne sait pas de quelle langue on devrait le traduire. Or, l'exégèse impose sa traduction, que la plupart des historiens acceptent, s'ils n'y souscrivent complètement ${ }^{12}$. Un choix de traduction, légitime en soi parmi d'autres choix possibles, fait jurisprudence au risque du contresens quand il finit par exclure d'autres traductions.

\section{Paresse du contresens}

L'envers de la question de l'erreur volontaire est donc la "paresse du contresens ». S'il est difficile d'imputer une part de volonté dans une erreur, sa répétition, une fois corrigée, ne prête plus de place au doute. La formule de La Bruyère à propos des amours supporte la paraphrase à propos des erreurs: "Les premières erreurs de traduction sont toujours les plus belles, les autres sont moins involontaires. " John Donne, le poète métaphysique et théologien anglais qui a contribué plus qu'aucun autre à cette remise à plat des traductions des textes bibliques, en a bien exposé l'enjeu dans son essai sur le suicide, le fameux Biathanatos, au début du XVII siècle: " [...] Il arrive très souvent qu'un Père, jouissant d'une grande réputation et d'une grande autorité en son temps, s'empare de quelque interprétation probable des Écritures puis, l'assimilant dans ses homélies, l'applique pour encourager ou détourner ses auditeurs au gré des occasions ou de leurs maladies, ou suivant ce que son époque exige. Ainsi se plaît-il à imaginer des insinuations figurées qu'il adapte à la musique de son style [...] en sorte que le sens, qui n'était que probable, devient nécessaire; et ceux qui lui succèdent ont plutôt apprécié son esprit qu'ils n'ont troublé le leur. Comme il arrive souvent, nous répugnons à changer, ou à abandonner, une pierre contrefaite pour la simple raison qu'elle est posée là ${ }^{13}$. " Avec Érasme, Richard Simon, Pierre-Daniel Huet et tant d'autres, John Donne a

11 M. Harl, La Bible en Sorbonne ou la revanche d'Érasme, Paris, Cerf, 2004, p. 291-292.

12 Voir P.-E. DaUzAT, "Judas trahi par la traduction: traduction, trahison et mort volontaire des évangiles à Sylvia Plath”, Revue française de psychanalyse, 2008/4, p. 973-989.

13 J. Donne, Biathanatos, trad. P.-E. Dauzat, Paris, PUF, 2001, $3^{\text {e }}$ partie, distinction V, section 7 . 
cerné avec acuité cette paresse du contresens ${ }^{14}$ dès le XVII siècle. Mais on aurait tort de croire que cette "paresse » est le propre d'une étape de la pensée en matière de traduction, celle des «belles infidèles ".

Évoquant la persistance des traductions fautives, Guillaume Gardascia pose trois questions centrales: "Comment un contresens grossier a-t-il, pu survivre pendant près de deux millénaires? Comment une fois dénoncé, peut-il rester ignoré pendant plusieurs lustres par la quasi-totalité des interprètes? Comment enfin, après une correction indiscutable et suffisamment diffusée, peut-on revenir si souvent à l'erreur ancienne ${ }^{15}$ ? » Sa réponse se limite à un constat. Dans le même ordre d'idées, l'historien Marc Bloch évoque le caractère approximatif du mot serf pour " désigner le chriépostnoï de l'ancienne Russie tsariste ». Mais il suggère une réponse en montrant les inconvénients du mot retenu par les historiens: "Un rapprochement plus malencontreux pouvait difficilement être imaginé. Là-bas, un régime d'attache à la glèbe, peu à peu transformé en un véritable esclavage; chez nous, une forme de dépendance personnelle qui, malgré sa rigueur, était très loin de traiter l'homme comme une chose dépourvue de tous droits: le prétendu servage russe n'avait à peu près rien de commun avec notre servage médiéval. Cependant, dire tout bonnement chriépostnö̈ ne nous avancerait guère. Car il a existé en Roumanie, en Hongrie, en Pologne et jusque dans l'Allemagne orientale, des types de sujétion paysanne étroitement apparentés à celui qui s'établit en Russie. Faudra-t-il, tout à tour, parler roumain, hongrois, polonais, allemand ou russe? Une fois de plus, l'essentiel échapperait, qui est de restituer les liaisons profondes des faits, en les exprimant par une juste nomenclature. » Et de conclure: "L'étiquette a été mal choisie. Une étiquette commune, surimposée, par conséquent aux noms nationaux au lieu de les copier n'en demeure pas moins nécessaire. Là encore, la passivité est interdite $^{16}$. " (C'est moi qui souligne.) Paresse et passivité font bon ménage.

Mais le problème conceptuel que rencontrent les historiens a son pendant dans la traduction dite poétique de textes qui, outre leur valeur littéraire, ont acquis par leur impact la valeur de document historique et constituent une source pour l'historien. Particulièrement révélateur est le cas d'Yves Bonnefoy, qui, au fond, refuse de traduire et, comme dit Jean Bollack, cherche à "se satisfaire dans une créativité plus libre ». Ainsi, quand il traduit le Winter's tale de Shakespeare (V, 1, 106 sq.), où il est dit que la beauté de Perdita (!) est telle que, would she begin a sect, might quench the zeal I of all professors else; make proselytes of who she but bid follow; le sens est par trois fois "faussé " quand zèle est rendu par ferveur, prosélytes par zélateurs, et les derniers mots par «n'importe qui ». Au nom du " poétique », et parce que le traducteur refuse d'être

14 L'expression est empruntée à Daniel Lys, "La paresse du contresens", Études théologiques et religieuses, 78, 2003, n 3, p. 309-320.

15 G. Gardascia, “Trahisons' bibliques: Nb 5,31 \& Lc 10,25-37”, Revue Biblique, n 3, juillet, 2005, p. 369-371.

16 M. Bloch, Apologie pour l'histoire, Paris, Armand Colin, 1974, p. 135. 
" lié par la traduction qui s'est faite dans la langue d'origine, et qui se déchiffre ${ }^{17}$ ", Shakespeare se trouve ainsi décontextualisé et le lecteur privé des références historiques, jusque dans le vocabulaire, qui lui permettrait de comprendre ce qu'il lit ${ }^{18}$. On voit bien que le constat de John Donne vaut pour tous les domaines sans qu'on puisse apporter de réponses définitives aux questions de Gardascia. Mais la " paresse du contresens " obéit aussi à d'autres ressorts: "La philologie biblique progresse, observe Daniel Lys, cependant que la langue de réception du texte traduit évolue. La combinaison des deux est génératrice de contresens. Par habitude paresseuse et reposante, on garde des interprétations dépassées [...]. " Souvent une traduction décide d'une interprétation autant qu'une interprétation dicte une traduction.

\section{Fausse pudeur et vrai contresens}

Si l'on délaisse le domaine religieux pour celui des études historiques, la problématique est la même. Cherchant à combattre le doute qui germe dans l'esprit de Bouvard et Pécuchet, l'abbé Jeufroy explique doctement: "Isaïe ne se dépouilla pas complètement, nudus, en latin, signifiant nu jusqu'aux hanches; ainsi Virgile conseille de se mettre nu pour labourer, et cet écrivain n'eût pas donné un précepte contraire à la pudeur! Ézéchiel dévorant un livre n'a rien d'extraordinaire; ne dit-on pas dévorer une brochure, un journal ${ }^{19}$ ? " Dans un roman désopilant, le grand érudit chinois Qian Zhongshu ne cesse de jouer sur les mots et évoque à maintes reprises les divers usages de la traduction; à propos d'une certaine Miss Bao aux formes avantageuses et aux vêtements plutôt moulants: les uns l'appellent la Vérité, parce qu'elle n'a rien à cacher, et les autres « Parcelle de Vérité » parce qu'elle n'était qu'à moitié nue ${ }^{20}$. La nudité d'Ésaïe est-elle une demi-vérité ou une vérité complète? Et s'il y a contresens de l'abbé Jeufroy, est-il ou non volontaire? Comment s'explique sa persistance? Dans leur Dictionnaire étymologique de la langue latine, Ernout et Meillet sont prudents: sub verbo "nudus », ils indiquent "peut-être ", à l'imitation du grec gumnos, "légèrement vêtu ", et renvoient, comme l’abbé Jeufroy, aux Géorgiques de Virgile; dans la Vulgate, il s'agit certainement d'un " néologisme de sens ». François d'Assise avait compris au sens littéral, quand il dansa nu pour être plus proche de la nature. Alors, traduction ou sollicitation, demi-vérité ou vérité?

17 Cf. J. Bollack, "Le traducteur", in Bollack, Poésie contre poésie. Celan et la littérature, Paris, PUF, 2001, p. 168-179, ici p. 179.

18 Ces remarques sont tirées de l'analyse probante de Éd. WiLl et Cl. OrRIEuX, "Prosélytisme Juif "? Histoire d'une erreur, Paris, Les Belles Lettres, 2004, p. 302, n. 53. Yves Bonnefoy s'est partiellement corrigé depuis en réintroduisant le "prosélytes ". Cf. Shakespeare, Le Comte d'hiver, Paris, Gallimard, Folio/Théâtre, 1996, p. 182.

19 Flaubert, Euvres, éd. A. Thibaudet et R. Dumesnil, Paris, Gallimard, Bibliothèque de la Pléiade, 1952, vol. 2, p. 936.

20 Qian Zhongshu, La Forteresse assiégée (1947), trad. S. Servan-Schreiber et Wang Lou, préface de L. BiAnCO, Christian Bourgois, 1987, p. 13. 
Dans son étude magistrale, Christianisme, tolérance sociale et homosexualité, John Boswell, à propos de l'histoire des pratiques homosexuelles a montré comment la pratique du contresens volontaire s'est imposée et a durablement entravé le développement des recherches ${ }^{21}$. Les erreurs volontaires ou manipulations portent autant sur l'édition des textes que sur leur traduction. Au-delà de l'Ovide moralisé, L'art d'aimer a été un terrain d'élection de ces pratiques. Boswell en relève un exemple parmi bien d'autres. Hoc est quod pueri tangar amore minus, soit « j'étais moins attiré par l'amour des garçons " devient: Hoc est quod pueri tangar amore nibil, qui justifie la notation marginale qu'Ovide n'était pas un sodomite. Selon Boswell, " des substitutions aussi grossières sont aisées à dénoncer et on a trouvé depuis des moyens plus subtils de déguiser les manifestations d'homosexualité. Il est courant de mettre au féminin des pronoms masculins au moins depuis que le petit-neveu de Michel-Ange imagina de rendre les sonnets du grand artiste plus acceptables [...] et cette ruse a gardé la faveur des universitaires même si aucune réputation morale n'était en jeu ".

Loin d'appartenir au passé, la controverse persiste autour des questions de traduction et de mauvaise foi. Le sens des inscriptions de Théra (VII ou VI ${ }^{\mathrm{e}}$ siècle avant J.-C), graffiti ou hommages évoquant parfois crument des pratiques homosexuelles, continuent ainsi de susciter la controverse et les accusations de contresens délibéré ou de sollicitation. Dans son histoire de l'homosexualité, Bernard Sergent ${ }^{22}$, s'en prend ainsi à Marrou qui, dans son histoire de l'éducation, rend oiphein par "saillir ${ }^{23}$ ": contresens "délibéré ", selon lui puisque oiphein n'est pas un mot grossier et ne se dit pas des animaux (cf. Chantraine) ${ }^{24}$. Autrement dit, Marrou aurait choisi par le simple biais de la traduction de dénaturer le sens de ces inscriptions pour en faire des salacités. Il est difficile, en l'occurrence, de faire la part de la polémique et celle de l'analyse savante: si les quelques pages de Marrou sur la parenté entre la culture homosexuelle chez les nazis et la culture homosexuelle chez les Grecs peuvent paraître problématiques aujourd'hui, rien n'autorise à y voir autre chose que le souci d'un disciple de Raymond Aron et précurseur de G. Mosse pour jeter des ponts entre le monde antique et les phénomènes contemporains. Il serait difficile de lui imputer un désir de tromper ${ }^{25}$.

21 J. Boswell, Christianisme, tolérance sociale et homosexualité, trad. A. Tachet, Paris, Gallimard, 1985, p. 40-44.

22 B. Sergent, L'homosexualité initiatique dans l'Europe ancienne, Paris, Payot, 1986, p. $34-35$ et 242 , note 41 .

23 H.-I. Marrou, Histoire de l'éducation dans l'Antiquité, Paris, Seuil, 1948; rééd. PointsSeuil, 1. Le Monde grec, 1981, p. $356 \$ 10$.

24 Cf. aussi K. Dover, in Homosexualité grecque, trad. S. SAÏD, La Pensée sauvage, 1982, p. 152, précisant que le mot n'est pas aussi argotique que "baiser " ou grossier que " foutre ", mais traduit ensuite par " fait l'amour".

25 Sur la question de l'imputation de l'erreur à l'ignorance, la facilité ou la malveillance, voir désormais E. BRONGERSMA, "The Thera inscriptions ritual or slander?", Journal of Homosexuality, 20 (4) 1990, p. 31-39. 


\section{Jérôme et le célibat}

L'erreur et la paresse se mêlent dans un autre domaine voisin où aucune correction traductologique ne saurait corriger la doxa. Dans la Vulgate, Jérôme insère un texte de l'histoire de Tobie qui differe du texte grec des Septante plus ancien. Lui-même avoue sa traduction hâtive. Quand le texte alexandrin fait dire au jeune Tobie, avant de s'unir à son épouse: "Et maintenant, ce n'est pas le plaisir que je cherche en prenant ma sœur ", Jérôme traduit: "Tu le sais, ce n'est pas par concupiscence que je prends ta sœur pour épouse, mais par amour de la postérité. » La traduction est l'occasion d'une moralisation. Et pour faire bonne mesure, Jérôme supprime la citation de la Genèse: "Il n'est pas bon que l'homme soit seul. " La traduction est ainsi l'occasion d'une double mutilation du texte, introduisant la procréation, absente de l'original, et supprimant la communauté des époux, pourtant explicitement mentionnée. Jérôme a eu d'autant plus d'influence que sa vision s'accordait avec une vision pessimiste de la sexualitée ${ }^{6}$, alors même que son interprétation repose sur une erreur de traduction.

Jérôme est aussi un des pères de l'idée que le célibat est supérieur au mariage. Il a lancé cette idée en interprétant Matthieu 13,8, la parabole du grain semé qui rapporte 30, 60 ou 100 selon la qualité de la terre. Jérôme en conclut que les célibataires consacrés rapportent 100, les veufs et les veuves qui ne se remarient pas offrent 60 et ceux qui donnent 30 sont les gens mariés ${ }^{27}$. Quand il traduit, commente Michel Rouche, Jérôme précise: tricesimum, sexagesimum et centesimum, non pas trigenta, sexaginta, centum. Et crée alors un contresens, car la bonne traduction donne " un trentième, un soixantième et un centième " (le taux d'intérêt de $1 \%$ étant celui que fixe le code théodosien pour le remboursement mensuel des prêts) : "Ce qui signifie [...], selon la traduction exacte, qu'un champ qui rapporte un centième rapporte en fait $12 \%$ par an, un soixantième $20 \%$ et un trentième $40 \%$ ! L'échelle de la mesure s'en trouve inversée ${ }^{28}$. "Augustin donnera raison à Jérôme ${ }^{29}$. Dès lors Pascal pourra parler du " mariage, la plus basse condition du christianisme».

Or le mariage deviendra un sacrement et prendra un caractère rigoureux du fait de la traduction latine du grec mystêrion in Eph. 5,32, à propos du mariage: après avoir invoqué la Genèse pour dire "les deux deviendront une chair ", Paul ajoute: "Ce mystère est grand: je dis par rapport au Christ et à l'Église. " Mystêrion n'a pas à l'origine le sens de rite initiatique. Dans son œuvre apologétique, cependant, Tertullien

26 Cf. M. Rouche, Le plaisir sexuel est-il un péché?, Paris, Éd. Le Prat, 1983, p. 42-69, notamment p. 47-48, sur les traductions hiéronymiennes.

27 Jérôme, Sur saint Matthieu, 13-23, tome I, Sources chrétiennes, Cerf, 1977, p. 272-274; voir aussi son $A d v$. Jovinianum, I, 3; et lettre 49 à Pammachius, 2, éd. Labourt, II, p. 121-122, où Jérôme se défend d'avoir jamais rabaissé le mariage.

M. Rouche, Sexualité, intimité et société sous le regard de l'Histoire, Paris, CLD, 2002, p. $80-81$.

29 Pour le point de vue de Jérôme, cf. sa lettre 49 à Pammachius. 
avait joué sur les mots avec son emploi spécifique du nom sacramentum qui, du serment des latins, devint une traduction du grec musterion, partant acquit ipso facto son sens spécifiquement chrétien ${ }^{30}$. Dès lors, quand la Vulgate traduira megas mystêrion par sacramentum magnum, elle créera le malentendu. Sans être totalement fausse puisqu'on peut comprendre sacramentum au sens de "profonde réalité sacrée ", plutôt qu'au sens technique reçu depuis son élaboration par la théologie médiévale ${ }^{31}$.

\section{Augustin et les marchands du temple}

Quand, au soir de sa vie, Augustin se penche sur son œuvre et compose des Retractationes, il avoue s'être trompé (Révisions, I, XII, 6). En affirmant que Jésus n'avait jamais rien fait par la violence, « je ne m'étais pas souvenu qu'il avait chassé du temple "les vendeurs et les acheteurs" avec un fouet " (Mc 11, 15-17, Mat 21,12, Lc 19,45). Cette " correction " découle assurément du point de vue qu'il avait développé et affûté au cours de la controverse donatiste, à savoir que la force est parfois nécessaire pour ramener les récalcitrants à des idées " justes ${ }^{32}$ ". À l'exemple d'Augustin, la Vulgate et les traductions consacrées laissent entendre que le fouet a été utilisé contre les marchands. Augustin se corrige, mais que corrige-t-il: une traduction ou une interprétation? Sa bévue philologique ou sa faiblesse doctrinale? Force est de constater que l'unanimité était loin d'être acquise en son temps. Dans un texte copte donnant l'évangile de Matthieu, récemment publié par Hans-Martin Schenke, Jésus n'effectue aucun acte violent lorsqu'il s'en prend aux vendeurs installés dans le Temple (Mt 21,12-13). Pour certains, ce détail important se lisait, non pas dans l'évangile de Matthieu, mais dans une forme archaïque de l'évangile de Jean qui, dans le manuscrit, aurait remplacé le texte matthéen primitif ${ }^{33}$. Pour d'autres, le problème est à la fois d'idéologie et de traduction: tout tourne autour d'une conjonction présente chez Jean 2,15. Le sens normal de la conjonction te kai est d'initier une liste, non pas de conti-

30 Fr. Refoulé, introd. à Tertullien, Le Baptême, Paris, Cerf, 1976, p. 66-70 et surtout R. Braun, Deus christianorum. Recherches sur le vocabulaire doctrinal de Tertullien, Paris, Études Augustiniennes, 1977, p. 435-443.

31 Sur l'évolution des termes sacramentum et mystêrion au début du christianisme, voir H.M. FÉRET, "Sacramentum Res dans la langue théologique de saint Augustin”, Revue des Sciences Philosophiques et Théologiques, 29, 1940, p. 219-223.

32 Voir E. Clark, "On not retracting the unconfessed", in J. D. Caputo et M. J. Scanlon, Augustine and Postmodernism. Confessions and Circumfession, Bloomington et Indianapolis, Indiana University Press, 2005, p. 222-243, ici p. 231 (trad. fr. in Jacques Derrida-Saint Augustin. Des confessions, trad. P.-E. Dauzat, Paris, Stock, 2006, p. 457). Peter Brown examine l'évolution d'Augustin de plus en plus partisan de la " coercition " à l'égard des donatistes in Religion and Society in the Age of Saint Augustine, New York, Harper \& Row, 1972, p. 260-278, 301-331.

33 M.-E. Boismard, "Jésus a-t-il usé de violence en chassant les vendeurs du temple?", Revue Biblique, 2003, vol. 110, $\mathrm{n}^{\circ}$ 1, p. 33-37. 
nuer une série commençant par « eux tous» (pantas), lequel peut renvoyer aux «vendeurs et changeurs » qui précèdent autant qu'aux « moutons et bœufs » qui suivent. Quant au verbe exebalen, il signifie simplement " renvoyer" et n'implique aucune violence ${ }^{34}$. $\mathrm{Au} \mathrm{VI}$ e siècle, le témoignage du Grec Cosmas Indicopleustès (v. 530), au livre III de sa Topographie chrétienne, 18-24 (144B-148A) dissipe toute ombre d'un doute: Jésus était armé d'un fouet (cf. Jn 2,15), mais "il ne frappa que les bêtes ", les " êtres privés de raison "; les autres, il les a "corrigés par la parole ${ }^{35}$ ». Barhadbšabba (mort en 628), donne l'origine de cette interprétation en exposant les raisons de l'inimitié de Rabbula pour Théodore de Mopsueste: «Rabbula montrait auparavant beaucoup d'amitié pour le célèbre Interprète et étudiait ses ouvrages. Mais étant allé à Constantinople pour assister au concile des Pères (381), il fut accusé de frapper les clercs; ayant répondu que Notre Seigneur frappa lui aussi quand il entra au temple, l'Interprète se leva et le réprimanda en disant: Notre Seigneur ne fit pas cela; aux hommes il adressa seulement la parole, disant: Ôtez-cela d'ici, et renversa les tables. Mais il fit sortir à coups de fouet les taureaux et les moutons ${ }^{36}$. " En l'occurrence, le témoignage de l'histoire et de la philologie ne suffisent pas à corriger une légende tenace. D'involontaire, peut-être, l'erreur est devenue volontaire, et s'est installée au prix d'une certaine paresse.

\section{En guise de conclusion}

À ceux qui se désoleraient de cette paresse et de ces manipulations, je rappellerai une curiosité, à savoir que la persistance de l'erreur de traduction ne change pas forcément quelque chose à la compréhension parce que la compréhension n'est pas liée ou pas subordonnée de manière univoque à l'erreur de traduction; là encore on peut trouver des équivalents dans le domaine profane. La traduction n'est qu'un moment, voire un outil, du travail de déchiffrement: elle est téléologique, sans être une fin en soi. Dans les Vexations de l'art Svetlana Alpers ${ }^{37}$ cite à plusieurs reprises une inscription de Vélasquez. À ses débuts à la cour (1625), où l'on reprocha au cheval qu'il avait peint dans un portrait du roi de contrevenir aux règles de l'art, Velázquez prit la mouche et refusa de corriger ses erreurs. Il préféra simplement effacer la majeure partie du tableau et remplaça sa signature par les mots suivants: Didacus Velazquius, Pictor Regis,

34 J. Lasserre, "Un contresens tenace", Cahier de la Réconciliation, Paris, octobre 1967, n 10, p. 7 sq. L'auteur relève 91 occurrences de cette conjonction: dans 86 cas, une traduction de même nature que " aussi bien que les moutons et les bœufs " serait impossible. Dans cinq cas, elle serait possible, mais tel n'a pas été le choix des traducteurs. La traduction de Jean 2,15 est donc la seule exception.

35 Cosmas Indicopleustès, Topographie chrétienne, éd. W. Wolska-Conus, Paris, Cerf/Sources chrétiennes, 1968, p. 458-459 (III, 22).

36 Cité in W. Wolska, La Topographie de Cosmas Indicopleustès. Théologie et science au $V^{e}$ siècle, Paris, PUF, 1962, p. 91-92.

37 Paris, Gallimard, 2008. 
expinxit, soit "Diego Velázquez, peintre du roi, l'a peint "; comprenant ce qu'il avait fait, l'historien d'art traduit expinxit tantôt par expunged tantôt par unpainted/erased ${ }^{38}$, l'a supprimé, " dé-peint " ou effacé, ce qui est très exactement un contresens: expingere veut dire peindre, représenter. Si le contresens flagrant ici commis n'a pas induit davantage en erreur les historiens d'art, c'est qu'ils disposaient d'une biographie d'un contemporain de Velázquez, Antonio Palomino, qui avait relaté l'épisode par le menu, permettant d'" effacer " (expingere) à son tour l'erreur de traduction.

Velázquez, s'exprimant par antiphrase, a provoqué un contresens. Il a cédé à l'ironie, laissant entendre une chose en en disant une autre: le procédé dont il a joué exploite la possibilité de contresens ou d'incompréhension, non pas dans le passage d'une langue à l'autre, mais dans sa propre langue. Comme dit Philippe Hamon: "L'ironie, d'un certain point de vue, pourrait donc être définie soit comme un "contresens", le contresens volontaire d'un énonciateur parlant "contre" un sens appartenant à autrui, soit comme un acte de réécriture, réécriture qu'opère le lecteur à partir du texte de l'auteur: il réécrit "beau" en "laid", "intelligent" en "stupide ${ }^{39 " . ~ " ~ L ' e ́ n o n c i a t e u r ~}$ Velázquez aurait commis un contresens volontaire; l'historien d'art se serait prévalu de sa qualité de lecteur pour réécrire et traduire le mot litigieux par son antonyme; et le traducteur, en l'occurrence moi-même, cédant à la peur de la rhétorique du contresens, a d'un seul geste rectifié le contresens, interdit la réécriture et effacé l'ironie en pensant que l'ironie pourrait échapper au critique de la traduction, qui aurait cru à une démarche inverse, avec un contresens de traduction avéré, mais qui a fait loi et qui est ensuite justifié, entre ironie, mauvaise foi et innocente paresse.

Pierre-Emmanuel DAUZAT

(traducteur et écrivain)

pierre.dauzat@wanadoo.fr

38 S. Alpers, The Vexations of Art, New Haven, Yale University Press, 2005, p. 160 et 257.

39 P. Hamon, L'Ironie littéraire. Essai sur les formes de l'écriture oblique, Paris, Hachette, 1996, p. 20-21. Et cette pratique de l'ironie n'est pas l'apanage de la modernité. Cf. également D. De Courcelles, "Entre la folie et les pleurs: la fonction de l'ironie lullienne dans le Desconhort (1295)", Estudis Romànics, Barcelone, Institut d'Estudis Catalans, 24, 2002, p. 139-148, ici, p. 142. 